\title{
On Period of Generalized Fibonacci Sequence Over Finite Ring and Tridiagonal Matrix
}

\author{
Yasemin Taşyurdu ${ }^{1 *}$, Zülküf Dilmen² \\ 1,2 Department of Mathematics, Faculty of Sciences and Art, The University of Erzincan, Erzincan, Turkey, \\ +9044622430 97, \\ ytasyurdu@erzincan.edu.tr \\ zlkf23dlmn@gmail.com \\ * Corresponding author \\ Received: 29th May 2016 \\ Accepted: $18^{\text {th }}$ November 2016 \\ DOI: http://dx.doi.org/10.18466/cbujos.302663
}

\begin{abstract}
In this study, $\left\{F_{n}\right\}$ Fibonacci sequence was defined over an arbitrary ring and its some properties are investigated. The terms of this sequence are derivated by Tridiagonal determinant of the matrix. It was shown that this sequence is periodic and their period is obtained. It was shown that the sequence obtained by reducing modulo $m$ coefficient and exponent of each Fibonacci sequence in arbitrary rings is periodic. It was seen that order of cyclic group generated with matrix $R=\left[\begin{array}{ll}a & 1 \\ b & 0\end{array}\right]$ is equal to the period of this sequence where $a, b$ are arbitrary elements of the ring. Also, the period of this sequence is compared with Wall number of Fibonacci sequence and it was shown that this period always was an even number.
\end{abstract}

Keywords - Fibonacci Sequence, Finite Ring, Period

\section{Introduction}

In modern science there is a huge interest in the theory and application of the Golden Section and Fibonacci numbers. Fibonacci numbers are one of the most well-known numbers, and it has many important applications to diverse fields suchas mathematics, computer science, physics, biology and statistics. The Fibonacci numbers $f_{n}$ are the terms of the sequence $0,1,1,2,3,5,8,13, \ldots$ where $f_{n}=f_{n-1}+f_{n-2}$ with the initial values $f_{0}=0$ and $f_{1}=1$. Fibonacci sequences and their related higher-order (tribonacci, $k$-nacci) sequences are generally viewed as sequences of integers.

Most of the study of Fibonacci sequences is done with groups. The notion of Wall number was first proposed by D. D. Wall [1] in 1960 and gave some theorems and properties concerning Wall number of the Fibonacci sequence. In the mid eighties, Wilcox extended the problem to abelian groups [2]. Knox proved that the periods of $k$-nacci ( $k$-step
Fibonacci sequences in dihedral groups were equal to $2 k+2$ [3]. Deveci, Karaduman and Campbell examined the behaviour period of the $k$-nacci sequence in the some finite binary polyhedral groups in [4]. However, very little is done with the rings. D. J. DeCarli [5] gave a generalized Fibonacci sequence over an arbitrary ring in 1970. This generalized Fibonacci sequence over an arbitrary ring $R$ is denoted by $\left\{F_{n}\right\}$ and defined by

$$
F_{n+2}=a F_{n+1}+b F_{n} \quad \text { for } \quad n \geq 0
$$

where $F_{0}=0$ (the zero of the ring), $F_{1}=I$ (the identity of the ring) and $a, b$ are arbitrary elements of ring $R$ [5]. Special cases of Fibonacci sequence over an arbitrary ring have been considered by $\mathrm{R}$. G. Buschman [6], A. F. Horadam [7] and N. N. Vorobyov [8] where this ring was taken to be the set of integers. O. Wyler [9] also worked with such a sequence over a particular commutative ring with identity. Taşyurdu and Gültekin obtain the period of generalized Fibonacci sequence in finite rings 
CBÜ Fen Bil. Dergi., Cilt 13, Sayı 1, 2017, 165-169 s

with identity of order $p^{2}$ by using equality recursively defined by $F_{n+2}=A_{1} F_{n+1}+A_{0} F_{n}$, for $n \geq 0$, where $F_{0}=0$ ( the zero of the ring), $F_{1}=I$ (the identity of the ring) and $A_{0}, A_{1}$ are generators elements of finite rings with identity of order $p^{2}$ $[10,11]$.

\section{Materials and Methods}

It is well known that the Fibonacci numbers: $f_{n}$ for $n=0,1, \ldots$ are defined by the Binet's formula as follows:

$$
f_{n}=\frac{1}{\sqrt{5}}\left(\alpha^{n+1}-\beta^{n+1}\right), \quad n=0,1, \ldots
$$

where $\alpha:=(1+\sqrt{5}) / 2$ and $\beta:=(1-\sqrt{5}) / 2$. The first few Fibonacci numbers are $1,1,2,3,5,8,13,21,34,55,89, \ldots$ The Fibonacci sequence $\left\{f_{n}\right\}$ is defined by

$$
f_{n}=f_{n-1}+f_{n-2}, \quad n=1,2, \ldots
$$

with the initial conditional $f_{-1}=0, f_{0}=1$. It is also widely know that the $f_{n}$ is related by the determinant of the special tridiagonal matrix of the form

$$
T_{n}=\left(\begin{array}{rrrrr}
1 & 1 & & & \\
-1 & 1 & 1 & & \\
& \ddots & \ddots & \ddots & \\
& & -1 & 1 & 1 \\
& & & -1 & 1
\end{array}\right) \epsilon R^{n X n}
$$

A sequence is periodic if, after a certain point, it consists only of repetitions of a fixed subsequence. The number of elements in the repeating subsequence is called the period of the sequence. For example, the sequence $a, b, c, d, b, c, d, b, c, d, \ldots$, is periodic after the initial element $a$ and has period 3. A sequence is simply periodic with period $k$ if the first $k$ elements in the sequence form a repeating subsequence. For example, the sequence $a, b, c, d, e, a, b, c, d, e, a, b, c, d, e, \ldots, \quad$ is simply periodic with period 5. [3]. The minimum period length of $\left(f_{i} \operatorname{modm}\right)_{i=-\infty}^{\infty}$ sequence is denote by $k(m)$ and is called Wall number of $m$ [1].

Theorem $2.1 k(m)$ is an even number for $n \geq 3$ [1].

Identity $2.2 f_{m+n}=f_{m-1} f_{n}+f_{m} f_{n+1}[12]$.

Identity $2.3 f_{m-n}=(-1)^{n}\left(f_{m} f_{n+1}-f_{m+1} f_{n}\right)$ [12].

From Identities 2.2 and 2.3 that

$$
f_{s+t}=f_{s-1} f_{t}+f_{s} f_{t+1}
$$

CBU J. of Sci., Volume 13, Issue 1, 2017, p 165-169

$$
f_{s-t}=(-1)^{t}\left(f_{s} f_{t+1}-f_{s+1} f_{t}\right) .
$$

If $f \equiv f_{t} \equiv 0$ then clearly $f_{s+t} \equiv 0$ and $f_{s-t} \equiv 0$. Hence all the zeros of $f(\bmod m)$ are evenly spaced throughout the sequence. Since $f(\bmod m)$ is periodic for any $m$ and $f_{0}=0$ it can be said that any integer will divide infinitely many Fibonacci numbers. The following are some immediate consequences of Wall number

$$
\begin{aligned}
f_{n} & \equiv f_{n+r . k(m)}(\bmod m) \\
f_{k(m)} & \equiv 0 \quad(\bmod m) \\
f_{k(m)-1} & \equiv f_{k(m)+1} \equiv f_{k(m)+2} \equiv 1 \quad(\bmod m)
\end{aligned}
$$

It will be often use the fact that if $f_{n} \equiv 0(\bmod m)$ and $f_{n+1} \equiv 1(\bmod m)$ then $k(m) \mid n[12]$.

D. J. DeCarli [5] gave a generalized Fibonacci sequence over an arbitrary ring in 1970 . Let $S$ be a ring with ideality I. consider the sequence $\left\{M_{n}\right\}$ of elements of $S$, recursively defined by

$$
M_{n+2}=a M_{n+1}+b M_{n} \quad \text { for } \quad n \geq 0,1,2, \cdots
$$

where $M_{0}, M_{1}, a$ and $b$ are abritrary elements of $S$ [5].

Definition 2.4 A special case of (2.1) equality is denoted by $\left\{F_{n}\right\}$ and defined by

$$
F_{n+2}=a F_{n+1}+b F_{n} \quad \text { for } n \geq 0
$$

where $F_{0}=0$ (the zero of the ring), $F_{1}=I$ (the identity of the ring) and $a, b$ are arbitrary elements of $R$ [5].

Theorem 2.5 If $F_{n+2}=a F_{n+1}+b F_{n}$, then $F_{n+2}=$ $F_{n+1} a+F_{n} b$ [5].

There is are a relation between the $\left\{M_{n}\right\}$ sequence and the $\left\{F_{n}\right\}$ sequence.

Theorem 2.6 $M_{n+r}=F_{r} a M_{n-1}+F_{r+1} M_{n} \quad n \geq$ $1, r \geq 0$ [5].

\section{Main Reults}

\subsection{Tridiogonal Matrix For Fibonacci Numbers in Finite Ring}

Definition 3.1.1 The Fibonacci sequence $\left\{F_{n}\right\}$ in the ring with identity is generated by a matrix $R_{2}$,

$$
R=\left(\begin{array}{ll}
a & I \\
b & 0
\end{array}\right), \quad R^{n}=\left(\begin{array}{cc}
F_{n+1} & F_{n} \\
b F_{n} & b F_{n-1}
\end{array}\right) .
$$


CBÜ Fen Bil. Dergi., Cilt 13, Sayı 1, 2017, 165-169 s

The Fibonacci sequence $\left\{F_{n}\right\}$ can be also computed using by the matrices. Let $A(n)$ be a family of tridiagonal matrices, as follows.

$$
A(n)=\left(\begin{array}{ccccc}
a_{11} & a_{12} & & & \\
a_{21} & a_{22} & a_{23} & & \\
& a_{32} & a_{33} & \ldots & \\
& & \ldots & \ldots & a_{(n-1) n} \\
& & & a_{n(n-1)} & a_{n n}
\end{array}\right)
$$

Theorem 3.1.2 The the determinants of $A(n)$ matrices are

$$
\begin{aligned}
\operatorname{det}(A(1)) & =a_{11} \\
\operatorname{det}(A(2)) & =a_{22} a_{11}-a_{21} a_{12} \\
& \ldots \\
\operatorname{det}(A(n)) & =a_{n n} \operatorname{det}(A(n-1)) \\
& \quad-a_{n(n-1)} a_{(n-1) n} \operatorname{det}(A(n-2))[13] .
\end{aligned}
$$

Thus, I can give the following theorem;

Theorem 3.1.3 For $n \geq 0$, if $F_{n}(a, b)$ is a tridiagonal matrix

$F_{n}(a, b)=$

$\left(\begin{array}{rrrlrrr}1 & -b & 0 & & 0 & 0 & 0 \\ 0 & a & -b & \cdots & 0 & 0 & 0 \\ 0 & 1 & a & & 0 & 0 & 0 \\ & \vdots & & \cdots & & \vdots & \\ 0 & 0 & 0 & & a & -b & 0 \\ 0 & 0 & 0 & \cdots & -b & a & -b \\ 0 & 0 & 0 & & 0 & 1 & a\end{array}\right)$

$F_{0}(a, b)=0$. Then, we have $\operatorname{det}\left(F_{n}(a, b)\right)=F_{n}$.

Proof . We will use the induction method on $n$. We can easely seen that

$$
\begin{aligned}
& \operatorname{det}\left(F_{1}(a, b)\right)=|1|=1=F_{1} \\
& \operatorname{det}\left(F_{2}(a, b)\right)=\left|\begin{array}{rr}
1 & -b \\
0 & a
\end{array}\right|=a=F_{2} \\
& \operatorname{det}\left(F_{3}(a, b)\right)=\left|\begin{array}{rrr}
1 & -b & 0 \\
0 & a & -b \\
0 & 1 & a
\end{array}\right|=a^{2}+b=F_{3} \\
& \operatorname{det}\left(F_{4}(a, b)\right)=\left|\begin{array}{rrrr}
1 & -b & 0 & 0 \\
0 & a & -b & 0 \\
0 & 1 & a & -b \\
0 & 0 & 0 & a
\end{array}\right|=a^{3}+2 a b=F_{4}
\end{aligned}
$$

We assume that

$$
\operatorname{det}\left(F_{n-1}(a, b)\right)=F_{n-1}
$$

CBU J. of Sci., Volume 13, Issue 1, 2017, p 165-169

$$
\operatorname{det}\left(F_{n-2}(a, b)\right)=F_{n-2} .
$$

So, we can write

$$
\begin{aligned}
\operatorname{det}\left(F_{n}(a, b)\right) & =a \operatorname{det}\left(F_{n-1}(a, b)\right)+b \operatorname{det}\left(F_{n-2}(a, b)\right) \\
& =a F_{n-1}+b F_{n-2} \\
& =F_{n}
\end{aligned}
$$

Thus, the proof is completed.

\subsection{The Generalized Fibonacci Sequences in Finite Ring Modulo $m$}

From Definition 2.4, we can write some of the terms of sequence $\left\{F_{n}\right\}$ as following:

$F_{0}=0$

$F_{1}=I$

$F_{2}=a I+b 0=a$

$F_{3}=a a+b I=a^{2}+b$

$F_{4}=a\left(a^{2}+b\right)+b a=a^{3}+2 a b$

$F_{5}=a\left(a^{3}+2 a b\right)+b\left(a^{2}+b\right)=a^{4}+3 a^{2} b+b^{2}$

$F_{6}=a\left(a^{4}+3 a^{2} b+b^{2}\right)+b\left(a^{3}+2 a b\right)$

$=a^{5}+4 a^{3} b+3 a^{2} b$

$F_{7}=a\left(a^{5}+4 a^{3} b+3 a^{2} b\right)+b\left(a^{4}+3 a^{2} b+b^{2}\right)$ $=a^{6}+5 a^{4} b+3 a^{3} b+3 a^{2} b^{2}+b^{3}$

Reducing the generalized sequence of coefficient and exponent of each Fibonacci sequence in finite ring with identity term by a modulus $\mathrm{m}$, we can get a repeating. Sequence denoted by

$$
\left\{F^{m}\right\}=\left\{F_{0}^{m}, F_{1}^{m}, \cdots, F_{n}^{m}, \cdots\right\}
$$

where $F_{i}^{m}=F_{n}(\operatorname{modm})$. Let $h F^{m}$ denote the smallest period of $\left\{F^{m}\right\}$, called the period of the generalized Fibonacci sequence in finite ring with identity modulo $m$.

Definition 3.2.1 The Fibonacci sequence $\left\{F_{n}\right\}$ in the ring with identity is generated by a matrix $R=\left(\begin{array}{ll}a & I \\ b & 0\end{array}\right)$,

$$
R^{n}=\left(\begin{array}{cc}
F_{n+1} & F_{n} \\
b F_{n} & b F_{n-1}
\end{array}\right)
$$

where $n \in \mathbb{Z}$.

Theorem 3.2.2 $\left\{F^{m}\right\}$ is a periodic sequence.

Proof. Let 
CBÜ Fen Bil. Dergi., Cilt 13, Sayı 1, 2017, 165-169 s

$S=\left\{a_{1} x+b_{1} y, a_{2} x+b_{2} y \mid 0 \leq a_{1}, a_{2}, b_{1}, b_{2} \leq m-\right.$

1). Then we have $|S|=\left(\mathrm{m}^{m}\right)^{2}$ being finite, that is, for any $i \geq j$, there exist natural numbers $i$ and $j$

$$
F_{i+1}^{m}=F_{j+1}^{m}, F_{i+2}^{m}=F_{j+2}^{m}, \ldots, F_{i+k}^{m}=F_{j+k}^{m}
$$

By definition of the generalized Fibonacci sequence in finite rings with identity we have that

$$
\begin{gathered}
F_{i}^{m}=a F_{i-1}^{m}+b F_{i-2}^{m} \\
F_{j}^{m}=a F_{j-1}^{m}+b F_{j-2}^{m} .
\end{gathered}
$$

If $F_{i+1}^{m}=F_{j+1}^{m}$ and $F_{i+2}^{m}=F_{j+2}^{m}$ then $F_{i+2}^{m}-F_{i+1}^{m}=$ $F_{j+2}^{m}-F_{j+1}^{m}$. So, $F_{i}^{m}=F_{j}^{m}$ where $F_{i+2}^{m}-F_{i+1}^{m}=F_{i}^{m}$ and $F_{j+2}^{m}-F_{j+1}^{m}=F_{j}^{m}$. Then we get $F_{i-1}^{m}=F_{j-1}^{m}$. As similarly, $F_{i-2}^{m}=F_{j-2}^{m}, \cdots F_{i-j}^{m}=F_{j-j}^{m}=F_{0}^{m} \quad$ which implices that the $\left\{F^{m}\right\}$ is a periodic sequence.

Example. For $m=2,\left\{F^{2}\right\}$ sequence is

$F_{0}=0$

$F_{1}=I$

$F_{2}=I a+0 b=a$

$F_{3}=a a+I b=a^{2}+b=I+b$

$F_{4}=a+b a+a b=a+2 b a=a$

$F_{5}=a a+I b+b b=a^{2}+b+b^{2}=I+b+I=b$

$F_{6}=b a+a b=I+I=0$

$F_{7}=0 a+b b=b^{2}=I$

We have $\left\{F^{2}\right\}=\{0, I, b, I+b, a, b, 0, I, \ldots\}$ and then repeat. So, we get $h F^{2}=6$.

Given a matrix $A=\left(h_{i j}\right)$ where $h_{i j}$ being Fibonacci sequence in the ring with real coefficients, $A(\bmod m)$ means that every entry of $A$ is modulo $m$, that is $\quad A(\operatorname{modm})=\left(\left(h_{i j}\right)(\operatorname{modm})\right)$. Let $\left\langle R_{2}\right\rangle_{m}=\left\{R_{2}^{\dot{I}}(\operatorname{modm}): i \geq 0\right\}$ be a cyclic group and $\left|\left\langle R_{2}\right\rangle_{m}\right|$ denote the order of $\left\langle R_{2}\right\rangle_{m}$ where $R_{2}^{\mathrm{i}}(\operatorname{modm})$ is reduction coefficient and exponent of each polynomial in $R_{2}^{\dot{1}}$ matrix modulo $m$.

Teorem 3.2.3 One has $h F^{m}=\left|\langle R\rangle_{m}\right|$.

Proof. To complete the proof, we will show that $h F^{m}$ is divisible by $\left|\langle R\rangle_{m}\right|$ and that $\left|\langle R\rangle_{m}\right|$ is divisible by $h F^{m}$. From Definition 3.2.1, we know that the Fibonacci sequence $\left\{F_{n}\right\}$ in the ring with identity is generated by a matrix $R$,
CBU J. of Sci., Volume 13, Issue 1, 2017, p 165-169

$$
R=\left(\begin{array}{ll}
a & I \\
b & 0
\end{array}\right), \quad R^{n}=\left(\begin{array}{cc}
F_{n+1} & F_{n} \\
b F_{n} & b F_{n-1}
\end{array}\right)
$$

where $n \in \mathbb{Z}$. Let $\left|\langle R\rangle_{m}\right|=s(m)$ where $s(m)$ is order of cyclic group generated with matrix $R$ according to modulo $m$. İt is clear that $o(R)=s(m)$ because of $\langle R\rangle_{m}=\left\{\left(\begin{array}{ll}a & I \\ b & 0\end{array}\right)^{i}(\bmod m): i \in \mathbb{Z}\right\}$. That is,

$$
\left(\begin{array}{ll}
a & I \\
b & 0
\end{array}\right)^{s(m)}(\operatorname{modm})=\left(\begin{array}{ll}
I & 0 \\
0 & I
\end{array}\right) .
$$

We will often use the fact that if $F_{h F} m \equiv 0(\bmod m)$ and $F_{h F^{m}+1} \equiv 1(\bmod m)$ the $s(m) \mid h F^{m}$ dir. $\langle R\rangle_{m} \mid h F^{m}$ is where $s(m)=\left|\langle R\rangle_{m}\right|$. Then we need to prove that $h F^{m}$ is divisible by $\left|\langle R\rangle_{m}\right|$. Let $h F^{m}=t$. We have already seen that $R^{t}=\left(\begin{array}{cc}F_{t+1} & F_{t} \\ F_{t} & F_{t-1}\end{array}\right)$ because $R^{t}=\left(\begin{array}{ll}I & 0 \\ 0 & I\end{array}\right)(\bmod m)$. So, $o(R)$ is divisible by $t$. We get that $\left|\langle R\rangle_{m}\right|$ is divisible by $t$ because $\left|\langle R\rangle_{m}\right|=o(R)$. That is, $h F^{m}$ is divisible by $\left|\langle R\rangle_{m}\right|$.

So, we get $h F^{m}=\left|\langle R\rangle_{m}\right|$.

The following are some immediate consequences of the Theorem 3.2.3 the using Identity 2.2 and Identity 2.3

$$
\begin{aligned}
F_{h F^{m}} & \equiv F_{h F^{m}+r . s(m)} \\
F_{s(m)} & \equiv 0 \quad(\bmod m) \\
F_{s(m)-1} & \equiv F_{s(m)+1} \equiv 1(\bmod m) .
\end{aligned}
$$

Theorem 3.2.4 $h F^{p}=p k(p)$ where $p$ is a prime number.

Proof. It is completed if it is that $\left|\langle R\rangle_{p}\right|$ is divisible by $p k(p)$ and that $p k(p)$ divisible by $\left|\langle R\rangle_{p}\right|$ because $h F^{p}=\left|\langle R\rangle_{p}\right|$ from Theorem 3.2.3 Let $\left|\langle R\rangle_{p}\right|=r$. So,

$$
\left(\begin{array}{ll}
a & I \\
b & 0
\end{array}\right)^{r}(\bmod p)=\left(\begin{array}{ll}
I & 0 \\
0 & I
\end{array}\right)
$$

where $R^{n}=\left(\begin{array}{cc}F_{n+1} & F_{n} \\ b F_{n} & b F_{n-1}\end{array}\right)$ for $R=\left(\begin{array}{ll}a & I \\ b & 0\end{array}\right)$. Also, $R^{p k(p)}=\left(\begin{array}{cc}F_{p k(p)+1} & F_{p k(p)} \\ F_{p k(p)} & F_{p k(p)-1}\end{array}\right)$. We can if $F_{p k(p)} \equiv$ $0(\bmod m)$ then $F_{p k(p)+1} \equiv F_{p k(p)-1} \equiv 1(\bmod m)$ where $k(p)$ denote the period of the Fibonacci sequence modulo $p$. So, we get $R^{p k(p)}=$ $\left(\begin{array}{ll}I & 0 \\ 0 & I\end{array}\right)(\bmod p)$. Thus Thus $p k(p)$ is divisible by $\left|\langle R\rangle_{p}\right|$. That is, $p k(p)$ is divisible by $h F^{p}$ where $h F^{p}=\left|\langle R\rangle_{p}\right|$. 
Then we need to show that $h F^{p}$ is divisible by $p k(p)$. Let $h F^{p}=s$. So, $F_{s} \equiv 0(\bmod m)$ and $F_{s+1} \equiv F_{s-1} \equiv 1(\bmod m)$. Thus,

$$
\left(\begin{array}{ll}
a & I \\
b & 0
\end{array}\right)^{s}(\bmod p)=\left(\begin{array}{ll}
I & 0 \\
0 & I
\end{array}\right)
$$

where $h F^{p}=\left|\langle R\rangle_{p}\right|=s$. So, $k(p) \mid s$ is where $k(p)$ denote the period of the Fibonacci sequence modulo $p$. That is, $h F^{p}$ is divisible by $p k(p)$. Therefore $h F^{p}=p k(p)$.

Theorem 3.2.5 $h F^{m}$ is an even number where $m$ is a integer.

Proof. İt has been shown that $h F^{m}=m k(m)$ in Theorem 3.2.4 If it is stated that $m k(m)$ is an even number then proof is completed. By Theorem 2.1 $k(m)$ is an even number and $m$ is an integer number for $m \geq 3$. Hence $m k(m)$ is always an even number. That is, $h F^{m}$ is an even number.

Table 1 shows some periods of sequence of coefficient and exponent of Fibonacci sequence in the with identity modulo $m$ by using $k(m)$.

Table 1: Periods of the Fibonacci sequence in the with identity modulo $m$

\begin{tabular}{|c|c|l|l|}
\hline$m$ & $k(m)$ & $h F^{m}$ & Results \\
\hline 2 & 3 & 6 & $h F^{2}=2 k(2)$ \\
\hline 6 & 24 & 144 & $h F^{6}=6 k(6)$ \\
\hline 10 & 60 & 600 & $h F^{10}=10 k(10)$ \\
\hline 15 & 40 & 600 & $h F^{15}=15 k(15)$ \\
\hline 17 & 36 & 612 & $h F^{17}=17 k(17)$ \\
\hline 131 & 130 & 17030 & $h F^{131}=131 k(131)$ \\
\hline 147 & 112 & 16464 & $h F^{147}=147 k(147)$ \\
\hline 257 & 516 & 132612 & $h F^{257}=257 k(257)$ \\
\hline 589 & 90 & 53010 & $h F^{589}=589 k(589)$ \\
\hline 610 & 60 & 36600 & $h F^{610}=610 k(610)$ \\
\hline 720 & 120 & 86400 & $h F^{720}=720 k(720)$ \\
\hline 944 & 696 & 657024 & $h F^{944}=944 k(944)$ \\
\hline
\end{tabular}

\section{Acknowledment}

The researchs of Y. Taşyurdu and Z. Dilmen were supported by Erzincan University Rectorship under "The Scientific and Research Project of Erzincan University", project no. FEN-A-2402150122.

\section{References}

[1] Wall, D.D.; Fibonacci series modulo m. Amer. Math. Monthly. 1960; 67(6), 525-532.

[2] Wilcox, H.J.; Fibonacci sequences of period $n$ in groups. Fibonacci Quart. 1986; 24(4), 356-361.

[3] Knox, S.W., Fibonacci Sequences in Finite Groups. Fibonacci Quart. 1992; 30(2), 116-120.

[4] Deveci, Ö.; Karaduman, E.C.; Campbell, M. On the knacci sequences in finite binary polyhedral groups. Algebra Colloq. 2011; 18(1), 945-954.

[5] De Carli, D.J., A Generalized Fibonacci Sequence Over An Arbitrary Ring. Fibonacci Quart. 1970; 8(2), 182184,198 .

[6] Buschman, R.G. Fibonacci Numbers, Chebyshev Polynomials, Generalizations and Difference Equations. Fibonacci Quart. 1963; 1(4), 1-7.

[7] Horadam, A.F. A Generalized Fibonacci Sequence. Amer. Math. Monthly. 1961; 68(5), 445-459.

[8] Vorobyov, N.N., The Fibonacci Numbers, translated from the Russian by Normal D. Whaland, Jr., and Olga A. Tittlebaum, D. C. Heath and Co., Boston, 1963.

[9] Wyler, O., On Second-order Recurrences. Amer. Math. Monthly, 72(5) (1965; 72(5), 500-506.

[10] Taşyurdu, Y.; Gültekin, İ. On period of Fibonacci sequences in finite rings with identity of order $p^{2}$. Journal of Mathematics and System Science. 2013; 3, 349352.

[11] Taşyurdu, Y.; Gültekin, İ. The Period of Fibonacci Sequences Over The Finite Field of Order $p^{2}$. New Trends in Mathematical Sciences. 2016; 4, 248-255.

[12] Renault, M. The Fibonacci Sequence Under Various Moduli" Master's Thesis. Wake Forest University. 1996.

[13] Cahill, N.D.; D’Errico, J.R.; Narayan, D.A.; Narayan, J.Y. Fibonacci determinats. College Mathematics Journal. 2002; 3(3), 221-225. 